\title{
PROCESSING OF HIGH NOISE DATA FROM INFRARED SENSOR IN HARSH ENVIRONMENT
}

D. Moskal, J. Martan*, V. Lang, M. Švantner

New Technologies Research Centre, University of West Bohemia, Univerzitni 8, 30614 Plzen, Czech Republic

\begin{abstract}
This article presents a new digital method, called Stamp method, for elimination of undesired noise data from a periodic IR signal. The Stamp method is a useful tool for statistical analysis of changes which appear in a noised input signal. One stamp is a statistical image of several consequence periods from a raw input signal. The Stamp method is a combination of equidistant transversal and differential filters, where useful output data is produced by subtraction of neighboring stamps. The corresponding digital Stamp filter formulation is provided with the Z-transform formalism. An application of the statistical Stamp method is presented on temperature changes extraction from a real noised IR (infra-red) raw signal from turning shaft in a power plant. The moving average and impulse-response filters were applied for final cleaning up of the data. The MATLAB short source code of stamp implementation is in the appendix.
\end{abstract}

Keywords: statistical methods, infrared radiometry, subtraction algorithm, IR-sensor signal processing

\footnotetext{
* Corresponding author. Tel: (+420) 377634718, Fax: (+420) 377634702, E-mail: jmartan@ntc.zcu.cz.
} 


\section{Introduction}

There are a lot of methods for analysis of changes in periodic signals of different nature sounds, mechanical vibrations, alternating electromagnetic processes, etc. [1]. A similar problem is solved when two or more signals of different natures are mixed together. However, the problem becomes more complicated in cases when the signal is mixed with thermal, random non-Gaussian or other noise [2-5]. This can occur in a case when a useful measured signal is at a same level or lower than a noise or other parasitic signal, which cannot be removed by a hardware method - by shielding, for example. The measured signal should be separated and the useful signal should be extracted by data processing in this case. When a useful signal is mixed with random noise, a statistical approach is one of the successful methods for data processing $[6,7]$.

In this article a new statistical Stamp method for raw periodical data processing is presented for solving the problems described. The Stamp method basics are presented with the mathematical approach and practical application of the method. A statistical approach plays a key role in the Stamp method. It does not provide an absolute value of the useful signal but it is focused on changes of the useful signal. Despite this limitation, the method is useful in a lot of cases where absolute values are not important, but possible changes of a useful signal can be an important factor and should be analyzed.

An example is a weak signal of an infrared (IR) sensor from a pulsed source demonstrated by a rotating shaft of a hydroelectric power plant generator with a temperature gradient or hot spots, where the useful signal is temperature variation and it is mixed with a high power electromagnetic emission. The measured signal is represented by the dotted line in Fig. 1. The amplitude of the electromagnetic field noise and undesired periodical signal is ten times bigger than useful signal, obtained by classical methods. Period of the undesired signal is equal to the period of the useful signal. Thus, classical methods, like the Butterworth 
passband signal processing filter, frequency response functions (FRF) with a fast Fourier transform (FFT), and a moving average filter (introduced by the "smooth" function in MATLAB, for example) [1, 8-11] are not strong enough for noise removal and cleaning up the raw measured data. An additional disadvantage of the frequency dependent methods mentioned is the necessity of a priori known characteristic of the useful signal for correct adjustment for filters. The data after processing using the described filters are shown in Fig. 1 by the solid line. The first quarter of the periods in the cleaned signal contains overlapped peaks and it is difficult to identify their nature: result of imperfections of the filters mentioned or real form of the useful signal. These results show that it is not easy to determine the position and size of temperature peaks from this evaluation. It is also not possible to determine the character of the changes in the useful signal: constant value or increasing from period to period and whether the increase is linear or exponential. To retrieve this information, the new method should be used for the data processing.

\section{Stamp method}

\subsection{Basic description}

The Stamp method is an evolution of subtraction algorithms with recursive logic. Subtraction algorithms are naturally used for cleaning up raw data from CCD matrices, video streams and statistical analyses of images [12 - 14]. A subtraction method is also used in measurement in a fusion plasma environment [15]. The subtraction algorithms are a good processing method for data, where an evaluation is not focused on absolute values of the data, but on local changes in a series of the data [16]. This method has more advantages when the undesired signal has a clean harmonic character and random noise, but the useful one has a more complex form. The new feature of the Stamp method presented is its statistical character. The fundamental idea of the Stamp method is based on two basic assumptions: 
I - the mean value of undesired signals and noise is the same in the whole studied region;

II - the useful signal is phase sensitive, but the random noise and the mentioned imperfections of mathematical methods are not phase sensitive.

The first assumption is acceptable in the case when undesired signals and noise are the same through whole processed region of the input data. In the case when the raw signal contains additional undesired signals or noise, which are changing by some function, then it is needed to apply special techniques, for example group analyses of the raw data [17]. The second assumption only underlines the fact, that influence of measurement system, imperfection of hardware or software, does not contain any information about period of measured signal. The application of these two assumptions of the Stamp method can be explained with the help of the logical scheme in Fig. 2. It is necessary to create one first stamp vector of incoming data as the first step. This stamp vector is formatted from $N$ periods from the first selected area of the raw signal (Fig. 2, Area 0). In this case the period value is a known constant, but in the other cases it is possible to use an autocorrelation function for period optimization (for example by maximal-minimal set-of). A sequence of the periods from this selected area is collected as the next step into the matrix $M^{0}$ :

$$
M^{0}=\left[\begin{array}{ccc}
P_{1,1}^{0} & \ldots & P_{1, K}^{0} \\
\ldots & P_{i, j}^{0} & \ldots \\
P_{N, 1}^{0} & \ldots & P_{N, K}^{0}
\end{array}\right]
$$

where $P_{i, j}^{0}$ is the value of the signal in period number $i$ in the data area 0 at the position $j$ from the beginning of this period; $N$ is the full number of periods in the selected area; $K$ is the full count of samples in one period. The full number of periods in the selected area $N$ has a limitation such that the abmodality should be smaller than $1 \%$ between the first and last period in the area. After the matrix $M^{0}$ is assembled, the statistical stamp $S^{0}$ is built as a vector, which contains the mean values of the data in columns of matrix $M^{0}$ :

$$
S^{0}=N^{-1} \cdot\left[\sum_{i=1}^{N} P_{i, 1}^{0}, \sum_{i=1}^{N} P_{i, 2}^{0}, \ldots, \sum_{i=1}^{N} P_{i, K}^{0}\right] .
$$


This equation represents the statistical stamp $S^{0}$ from the first area in the input signal. This starting stamp $S^{0}$ plays a key role in the presented method for providing the subtraction algorithm. The next stamp $S^{1}$ should be prepared from the next selected area from the raw signal in the same way. The resulting output of the stamp method returns only statistical image about changes of the signal in data region and regression of the noise stay to be unfinished. For full finished stamp method processing it is needed to apply additional classical methods, as Butterworth filter [11] and smooth function [1].

\subsection{Digital filter Stamp}

The convolution of input data in the one output value of the stamp is provided by a summation of all equidistant values from the input signal:

$$
y(j)=N^{-1} \cdot(x(j)+x(j-1 \cdot K)+\cdots+x(j-N \cdot K))=N^{-1} \cdot \sum_{n=0}^{N} x(j-n \cdot K),
$$

where $y(j)$ is the output data in the stamp and $x(j)$ is the input data value. In this way, every sample of the stamp is a statistical image of the equidistant samples from the whole area. This is a basic difference between the presented Stamp method and corresponding transversal digital filters. The next step of the Stamp method is providing subtraction between two neighbor stamps:

$$
y_{s}(j)=N^{-1} \cdot\left(\sum_{n=0}^{N} x(j-n \cdot K)-\sum_{n=0}^{N} x(j-N \cdot K-(n+1) \cdot K)\right) .
$$

Here the impulse response of the system is $N^{-1}$. The system is stable, because the $N$ is not zero and is not infinite. One period contains more than one sample [18, p. 174]. The subtraction procedure, which is presented in Eq. (4), returns data about changes of the signal between two wide areas. These areas contain several periods, and as a result, the output data has a statistical character.

The block diagram of the subtraction algorithm with Z-transform indexes is shown in Fig. 3. On this diagram, the process of filtration is presented only for two samples from neighbor 
stamps. In fact, it is a combination of two filters - equidistant transversal and difference filters. In Fig. 4 there is shown a frequency response of the Stamp filter determined by transfer function with $\mathrm{N}=200$ (number of periods) and $\mathrm{K}=1400$ (number of samples in a period). This filter has a lowpass characteristic for all harmonics with a period not divisible with the duration of the stamp. The corresponding transfer function of this filter has the form:

$$
H(z)=N^{-1}\left(\sum_{n=0}^{N} z^{-n \cdot K}-\sum_{n=0}^{N} z^{-N \cdot K-(n+1) \cdot K}\right) .
$$

This transfer function has not denominator, because the filter has not recursive connections.

There are a lot of other possibilities for filtering of the raw data. For example moving average filtering of the data by a $(n+1 / 2)$ periods, special designed band pass filter or symmetrisation of the filter. These methods are well known and useful tools for cleaning of raw data and can be used in combination of the Stamp method, especial with fine tuning of filters parameters. But the main difference of the Stamp filter from these mentioned filters is statistical data about changes of the input signal. The stamp method can be described as a hybrid of the subtraction and equidistant transversal filters.

\section{Application of the Stamp method for a real IR signal}

The Stamp method application procedure and functionality is demonstrated on an example of temperature measurement by a non-contact infrared sensor.

\subsection{Experiment description}

The experiment was focused on temperature changes measurement of a steel shaft, which was a part of a hydroelectric power plant generator. The measurement was performed within a diagnostic period of the system, which could detect possible problems and prevent production shut-downs or a damage of components of the system. Such a complication could be also an inhomogeneity of the shaft circumference temperature distribution. Therefore, objectives of 
the experiment were to measure temperature changes and temperature profile of the shaft along its circumference during its operation.

The shaft diameter was $1100 \mathrm{~mm}$ and its circumferential speed reached up to $7 \mathrm{~Hz}$ (about 430 revolutions per minute), which meant a shaft surface velocity approximately $25 \mathrm{~m} . \mathrm{s}^{-1}$. Contact temperature measurement using thermocouples was not possible, thus, a non-contact temperature measurement using an infrared-sensor was performed. A PVI-3TE-6 infrared sensor (VIGO system) with 3-level Peltier cooling and a MIPDC-F-5 control unit (control, power supply and preamplifier) were used for the temperature measurement. A shaft surface under the IR sensor was painted by a high-emissivity painting for homogenization and increasing of the measured surface emissivity, which could enhance measurement accuracy. Individual revolutions of the shaft were indicated by an induction ABS type sensor. The measurement was performed at an accessible position of the shaft, which was near a power generator and other power equipment components. The surroundings of the sensors and measurement devices location (working space of the power generator) were therefore influenced by a high electro-magnetic field. As the working space of the generator was not accessible during its operation, a data acquisition device (Dewetron measurement system) was located outside this area and connected with the sensors by wires. The scheme of the measurement configuration and the photography of the used IR sensor are shown in Fig. 5. The sampling frequency was $10 \mathrm{kHz}$ and the overall measurement time was about 2 hours. The data analysis was performed by a MATLAB numerical system.

\subsection{Converting and subtraction}

The application of the Stamp method and results are presented for an example of the processing of real measured data from the infrared sensor. In this case, it is necessary to convert a heat radiation value to absolute temperature with the calibration function $T_{j}^{0}=$ 
$f_{T}\left(S_{j}^{0}\right)$. The subtraction of temperature points of the stamp $S^{0}$ from the corresponding points of the next stamps $S^{1}$ causes suppression of the undesired periodic data (according to the first assumption of the Stamp method) and enables the obtaining of temperature value changes. In this way, the changes of temperature distribution between the selected areas can be found as stamp subtraction matrix $D$ :

$$
D=\left[\begin{array}{c}
D^{1} \\
\cdots \\
D^{r} \\
\cdots \\
D^{R}
\end{array}\right]=\left[\begin{array}{c}
f_{T}\left(S^{1}\right)-f_{T}\left(S^{0}\right) \\
\cdots \\
f_{T}\left(S^{r}\right)-f_{T}\left(S^{0}\right) \\
\cdots \\
f_{T}\left(S^{R}\right)-f_{T}\left(S^{0}\right)
\end{array}\right]
$$

where $r$ is the number of the analyzed area and $R$ is the total number of areas. The resulting matrix $D$ contains data about changes of the useful signal (Fig. 6). After the Stamp method processing, amplitudes of the undesired signals and the noise become ten times smaller than their amplitudes in the raw signal. This allows using standard filtering methods for extraction of the useful signal.

\subsection{Final clearing up}

The data processed by the Stamp method still contains irregular peaks and noise from the undesired signals which were not cleaned up by the processing. After the Stamp processing, it is therefore suitable to perform a final cleaning up of matrix $D$ by using the classical Butterworth filter [11] and smooth function [1]. However, the final cleaning up is in this case done without applying of FRF [9], which requires to define user-specified undesirable harmonics frequencies.

Consecutive applications of the Butterworth filter [11] and smooth function [1] for stamp subtraction vectors $D^{r}$ returns cleaned stamps $B^{r}$ :

$$
B^{r}=f\left(D^{r}, \text { Butterworth, Smooth }\right)
$$


The result of this cleaning process (Eq. (7)) is a sequence of fractured vectors $B^{r}$ (Fig. 7). Comparison of the signal after the Stamp method processing to the raw signal is shown in Fig. 8. The raw signal is in voltage (left axis) and the Stamp result is a change of voltage (right axis). It can be seen that the signal after Stamp processing is much lower than the raw signal containing noise and undesired signals. The Stamp processed data have much more noticeable peak (triangular marker in Fig. 8), which indicates clearly position on shaft where maximum temperature change occurs.

Imperfection of the moving average filter results in appearance of additional peaks at the edges of the processed data $[19$, p. 432] (Figs. 7 and 8, at the edges of the stamp). The Stamp method overcomes this problem by the connection of several stamps from the whole area into a vector $D S$ :

$$
D S=f_{T}\left(S^{1}\right)-f_{T}\left(S^{0}\right), \ldots, f_{T}\left(S^{r}\right)-f_{T}\left(S^{0}\right), \ldots, f_{T}\left(S^{R}\right)-f_{T}\left(S^{0}\right) .
$$

The undesirable edge effects become insignificant (Fig. 9) if the Butterworth filter and smooth function are applied on the $D S$ vector.

\section{Stamp method properties}

A useful feature of the Stamp method is the possibility to construct stamps from more periods. The stamp time length is then $K \cdot W$ and the stamp is expressed as:

$$
S^{r}=N^{-1} \cdot\left[\sum_{i=1}^{N} P_{i, 1}^{0}, \sum_{i=1}^{N} P_{i, 2}^{0}, \ldots, \sum_{i=1}^{N} P_{i, K}^{0} \cdot W\right],
$$

where $K$ is the length of one period, $W$ is the number of periods and $r$ is the number of the area. A long sequence vector $D L$ describes temperature changes in the signal with several periods. It is obtained by subtraction of two consecutive stamps in a temperature format:

$$
D L=f_{T}\left(S^{r+1}\right)-f_{T}\left(S^{r}\right) \text {. }
$$

The result of the cleaning process (Eq. (7)) of the vector $D L$ (Fig. 10, solid line) has not so important incorrect values at the edges in comparison to the short $B^{5}$ vector (Fig. 7 and 
Fig. 8). Moreover, the longer stamp $D L$ makes an autocorrelation for scale between thermal maximums in the processed signal. This means that every maximum in sequence (8) is the result of subtraction between equally spaced points in the signal. It gives a possibility to determine the character of temperature changes: linear, exponential or some other function. The example in Fig. 10 (solid line, temperature changes analyzed by the IR sensor) shows that the peaks have nearly the same maximum values. This means that the temperature changes have a linear character in the analyzed region of the signal.

The vector $D L$ in Fig. 10 has four local maximums. These maximums can be based on the real measured pulsed IR signal or can be caused by imperfections of the applied mathematical methods. The solution of this issue is in the second assumption of the Stamp method. If the input data are phase shifted, then the local maximums in the output vector $D L_{\text {shift }}$ must be shifted as well after applying of the Stamp method. It can be tested using, for example, a delay of $K / 8$, which is used for increasing the index of samples in the IR-sensor source data $x$ :

$$
y(j)=f\left(x\left(j+\frac{K}{8}\right)\right)
$$

where $x$ is the vector of IR-sensor raw data, $j$ is the index of the sample in vector $x$, and $y$ is the new data vector with a shifted index. The resulting vector $D L_{\text {shift }}$ is obtained by applying the Stamp method and the cleaning process to the vector $y$. The $D L_{\text {shift }}$ vector contains the same edge imperfections and local maximums as the vector $D L$. These maximums are shifted at positions exactly specified by the delay of $K / 8$ (Fig. 10, dotted line). This confirms the correlation between the positions of the extremes and indicates that their source is really the useful signal in the input data.

If changes of absolute values of the signal are required, then it is possible to construct a sequence of mean values from stamps. Consecutive stamps mean values and their sequence make it possible to analyze the general character of temperature changes (Fig. 11). This is an 
advantage of the Stamp method as well, because the influence of the noise and undesired data decreases as their mean value in one stamp tends to zero.

There are several other possibilities for future implementation of the described Stamp method. For example, it is possible to obtain the start stamp $S^{0}$ from a middle part of a measured interval of data or from statistical analyses of several areas.

\section{Summary}

The described Stamp method can be summarized into seven steps:

1. Dividing of the input raw data into several equivalent areas;

2. Dividing of every area into several periods;

3. Obtaining of one stamp as a mean value of the data from all periods in one area;

4. Obtaining of next stamp as a mean value of the data from all periods in the next area;

5. Conversion of every stamp to the correct physical units (e.g. temperature);

6. Subtraction of the stamps;

7. Final processing by the Butterworth filter [11] and smooth function [1].

\section{Conclusion}

A new method for treatment of measured data, called Stamp method, was presented in this paper. The method can be used for extraction of helpful data from a periodic signal highly loaded with random noise and undesired data from sources with a different nature. This method is simple and straightforward, because it is applied directly to the raw data without transformation to complex or trigonometric forms [20]. Additionally, the algorithm does not require any a priori known spectrums for the filtering. This makes the presented method a useful tool for power plant control, metal industry, meteorology, astronomy and anywhere for processes where continuous monitoring is required. 
The Stamp method is a combination of equidistant transversal and differential filters, where useful output data is produced by subtraction of neighboring stamps. One stamp is a statistical image of the several consequence periods from a raw input signal. The subtraction of neighboring stamps returns statistical data about changes in the useful signal. This statistical character is an advantage of the presented Stamp method. The corresponding digital Stamp filter formulation was provided with the Z-transform formalism.

Application of the Stamp method in both configurations (the short or the long stamp) enables exact positioning of the local maximum in one period. It allows, for example, an exact determination of a hot place on a rotating shaft and the character of its temperature increase in time even for harsh conditions with a lot of electromagnetic perturbations. The method was tested on a real data from IR sensor temperature measurement in a power plant. The results proof that the presented new method is useful and gives good results in correlation with predetermined assumptions.

\section{Acknowledgements}

The result was developed within the CENTEM project, reg. no. CZ.1.05/2.1.00/03.0088, cofunded by the ERDF as part of the Ministry of Education, Youth and Sports OP RDI programme and, in the follow-up sustainability stage, supported through CENTEM PLUS (LO1402) by financial means from the Ministry of Education, Youth and Sports under the ”National Sustainability Programme I. We thank Ing. Pavel MAUTNER, Ph.D. (University of West Bohemia, Department of Computer Science and Engineering) for his comments that greatly improved the manuscript. 


\section{References}

[1] S. W. Smith, The Scientist and Engineer's Guide to Digital Signal Processing (2nd ed., California Technical Publishing, San Diego, pp. 261-350, 1999).

[2] E. Ollila, IEEE Signal Process. Lett. 15, 841 (2008).

[3] Y.Sun, P. Willett, and P. F. Swaszek, IEEE Signal Process. Lett. 11, 189 (2004).

[4] V. Syrjala, M. Valkama, L. Anttila, T. Riihonen and D. Korpi, IEEE Transactions on Wireless Communications. 13, 2977 (2014).

[5] A. Sahai, G. Patel, C. Dick and A. Sabharwal, IEEE Transactions on Vehicular Technology. 62, 4494 (2013).

[6] A. Kowalczyk, R. Hanus, and A. Szlachta, Metrol. Meas. Syst. 18, 335 (2011).

[7] B. Stawarz-Graczyk, D. Dokupil, and P. Flisikowski, Metrol. Meas. Syst. 17, 95 (2010).

[8] A. V. Oppenheim, R. W. Shafer, and J.R. Buck, Discrete-time signal processing (2nd ed., Prentice Hall, New Jersey, pp. 824-826, 1998).

[9] C.F. Nelson and T.G. Carne, Removing undesired periodic data from random vibration data. In: IMAC XXV: Conference \& Exposition on Structural Dynamics - Celebrating 25 Years of IMAC, Orlando, Florida (2007).

[10] T.W. Parks and C.S. Burrus, Digital Filter Design (John Wiley \& Sons, New York, pp. 209-213, 1987).

[11] www.mathworks.com/help/signal/ref/butter.html (April 2015).

[12] M.V. Newberry, Signal-to-noise considerations for sky-subtracted CCD data. Astronomical Society of the Pacific, Publications (ISSN 0004-6280) 103, 122 (1991).

[13] C.R. Jung, IEEE Trans. Multimedia 11, 571 (2009).

[14] J. Lee and M. Park, Sensors 12, 12279 (2012).

[15] P. J. Fimognari, D. R. Demers, X. Chen and P. M. Schoch, Rev. Sci. Instrum. 85, $11 \mathrm{D} 849$ (2014). 
[16] Y. Benezeth, P. M. Jodoin, B. Emile, H. Laurent, and C. Rosenberger, Review and evaluation of commonly-implemented background subtraction algorithms. In 19th International Conference on Pattern Recognition (ICPR 2008). doi: 10.1109/ICPR.2008.4760998.

[17] X. Shen, H. Zhang, Y. Xu and S. Meng, IEEE Sensors Journal, 16, 7, pp. 1998-2003 (2016).

[18] E. C. Ifeachor, B. V. Jervis, Digital Signal Processing. A Practical Approach. (Harlow, England; New York: Prentice Hall, pp. 933, 2002).

[19] S. Brandt, Data Analysis. Statistical and Computational Methods for Scientists and Engineers. (Springer, New York, pp. 432, 1999).

[20] M. Richardson, Sounds and Vibration Magazine 12, 40 (1978). 


\section{Figure captions:}

1. Real signal from IR sensor, which contains noise and an undesired periodic signal from a powerful electromagnetic field. Dotted line - raw data with signals from several sources with different natures. Solid line - data about temperature of object after applying Butterworth filter, FRF and smooth function for the noised IR signal.

2. Scheme of stamp gathering from six areas with 10 periods in each area.

3. Block diagram of subtraction algorithm with Z-transform indexes.

4. Frequency response of the Stamp filter determined by transfer function with $\mathrm{N}=200$ (number of periods) and $\mathrm{K}=1400$ (number of samples in the period)

5. Scheme of the measurement configuration and IR sensor picture

6. Data about temperature changes between start and fifth stamps. The time length of one stamp is equal to one period.

7. Data about temperature changes obtained from the fifth area of data (vector $B^{5}$ ) by the Stamp method with consecutive applications of Butterworth and smooth filtering (incorrect maximums from the smooth method appear on the edges of the graph).

8. Comparison of the raw signal (dotted line, left axis) with the signal after the Stamp method processing (solid line, right axis). The triangular marker indicates maximum temperature change in the processed area $\left(\operatorname{vector} B^{5}\right)$.

9. Data about temperature changes in a region with four areas joined together obtained by the Stamp method with consecutive applications of Butterworth and smooth filtering.

10. Temperature changes in a widened area with consecutive applications of the Stamp method (long stamp), Butterworth and smooth filtering (solid line - no phase shifted; dotted line - shifted by K/8). 
11. Real signal from IR sensor (dotted line, left axis) and mean temperature value for the same time interval (solid line, right axis) gathered as mean values from consecutive stamps. 


\section{Appendix}

Main M-File (generator_and_stamp.m)

$\%$ Cleaning raw data from sources with different natures.

$\%$ At the very start the workspace will be cleared

clear all; close all; clc;

$\%$---- Process control interface ------------ $\%$

stBar = waitbar $(0$, 'Initializing ...'); \% Start wait bar for process view

1 Stp $=5 ; \quad \%$ Number of logical steps

$\%$----- Base settings for signal generator ------------------------------\%

discret $=10000 ; \quad \%$ Rate at which time-history samples are acquired $[\mathrm{Hz}]$

$\mathrm{bFr}=50 ; \quad \%$ Base frequency for electromagnetic field

$\mathrm{rFr}=7.15 ; \quad \%$ Frequency of IR-signal (for useful data)

$\mathrm{sL}=600 ; \quad \%$ Signal length [sec]

$\mathrm{sFr}=300 ; \quad \%$ Phase shifting for useful pulses

$\mathrm{nH}=7 ; \quad \%$ Number of harmonics in undesired spectrums

1 Stp $=1$ Stp $+\mathrm{nH} ; \quad \%$ Number of logical steps with harmonics buildings

$\mathrm{nP}=1 / 5 ; \quad \%$ Noise power

$\%$---- Filters settings ----------------- $\%$

$\mathrm{B} 1=2 ; \quad \%$ Order of Butterworth filter

B2 $=0.01 ; \quad \%$ Cutoff frequency for Butterworth filter

$\mathrm{Sm}=195 ; \quad \%$ Number of points used for moving average filter

As $=1000 ; \quad \%$ Area size in periods for stamp method

\%----- Timing -----------------------------------------------------------\%

dTime $=1 /$ discret; $\quad \%$ Time step

time $=(0:$ dTime $: s L) ; \%$ Time vector with sL length

$\%$---- Constants for spectrums --------------------------------\%

ampBe $=2.201 ; \quad \%$ Pre-exponential factor for electromagnetic

$\%$ field spectrum

powerBe $=-0.009 ; \%$ Argument of the exponential for electromagnetic field $\%$ spectrum

ampRe $=0.209 ; \quad \%$ Pre-exponential factor for IR-signal spectrum

powerRe $=-0.054 ; \%$ Argument of the exponential for IR-signal spectrum

$\%$---- Pulse characterization -------------------------\%

pulseC $1=7.541 \mathrm{e}-6$; $\%$ First coefficient for IR-signal increasing function

pulseC2 $=0.1 ; \quad \%$ Second coefficient for IR-signal increasing function

pulse $=($ pulseC $1 *$ time + pulseC2 $) . * \ldots$

$\left(\cos \left(2 * \mathrm{pi}^{*} \mathrm{rFr} *\right.\right.$ time $\left.\left.+\mathrm{sFr}\right)>0.99\right)$;

$\%$----- Creation of raw data and noise signal to demonstrate technique ----\%

raw $=\mathrm{nP}^{*}(\operatorname{rand}((\mathrm{sL} *$ discret $) \ldots \%$ Preallocate memory

$+1,1)-0.5)^{\prime}$;

$\mathrm{bFrH}=\mathrm{bFr} ; \quad \%$ Start harmonic of base frequency

$\mathrm{rFrH}=\mathrm{rFr} ; \quad \%$ Start harmonic for IR-signal frequency

for $\mathrm{kk}=1: \mathrm{nH} \quad \%$ Add periodic data to the random data

ampB $=$ ampBe*exp(powerBe*kk); \% Imitation of spectrum

ampR $=$ ampRe*exp(powerRe*kk); \% Imitation of spectrum of IR-signal

waitbar(kk/1Stp,stBar,(['Building harmonics ',num2str(kk)])); 


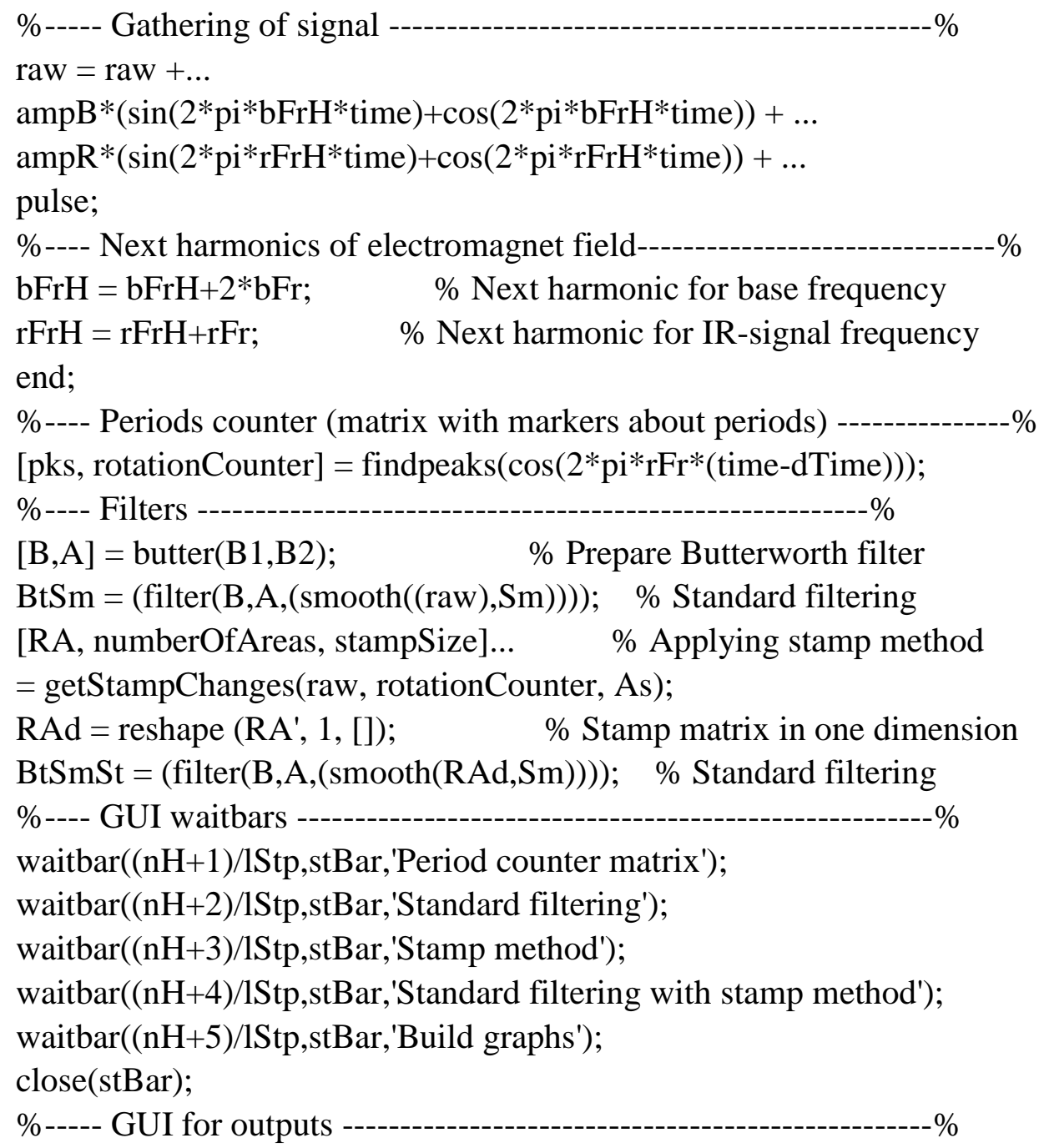

FigHandle = figure;

set(FigHandle, ... \% Position of figure

'Position', [100, 100, 1049, 895]);

subplot $(3,1,1) ; \quad \%$ Range for signal cleaned without stamp method plot(raw); $\quad \%$ Build signal cleaned without stamp method

title('Raw signal'); $\quad \%$ Caption for graph

xlabel('Samples'); \% Caption for abscissa

ylabel('U (V)'); \% Caption for ordinate

$x \lim ([0$ (numberOfAreas-1)...\% Diagrams accordance

*stampSize]);

subplot(3,1,2); $\quad \%$ Range for signal cleaned without stamp method

plot(BtSm); $\quad \%$ Build signal cleaned without stamp method

title(['Signal cleaned'... \% Caption

'by Butterworth filter and moving average filter']);

xlabel('Samples'); \% Caption for abscissa

ylabel('U (V)'); \% Caption for ordinate

$x \lim ([0$ (numberOfAreas-1)...\% Diagrams accordance

*stampSize]);

subplot $(3,1,3) ; \quad \%$ Range for signal cleaned with stamp method

$\operatorname{plot}(\mathrm{BtSmSt}) ; \quad \%$ Build signal cleaned with stamp method 


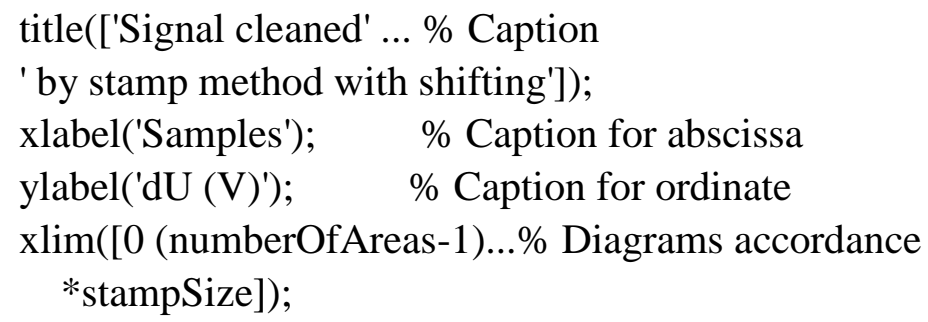

\section{M-File with core of Stamp method (getStampChanges.m)}

$\%$ Returns differential value from several periods by stamp method function [matrixOfChanges, numberOfAreas,stampSize]=... getStampChanges(inputSignal,rotationCounter, areaSize) $\mathrm{nR}=$ length(rotationCounter); $\%$ Number of periods numberOfAreas $=$ floor $(\mathrm{nR} / \mathrm{areaSize}) ; \%$ Number of areas $\%$ Minimal size of stamp $\%$ (because IR-signal frequency is $\%$ not multiplied for sample frequency) stampSize $=\min ($ rotationCounter $(:, 2$ :end $)$-rotationCounter(:,1:end-1) ; \%--- Create stamps by matrix M (matrixOfChanges) ------------------\% matrixOfChanges $=$ zeros $($ numberOfAreas $-1, \ldots \%$ Preparing empty matrix stampSize);

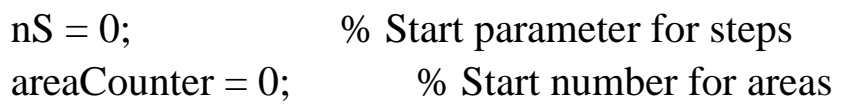

$\%$ The core of stamp method

if $n>1$

$$
\text { stamp }=\text { mean }(\text { area }) ; \quad \% \text { Get mean value of stamp }
$$

$\%$ Conversion to temperature

$\%$ should be here

matrix OfChanges(areaCounter,:) $=\ldots \%$ Get differential value stamp - stamp0;

else

stamp $0=$ mean $($ area $) ; \quad \%$ Get mean value of stamp

end;

areaCounter $=$ areaCounter $+1 ; \quad \%$ Counter for areas end; 


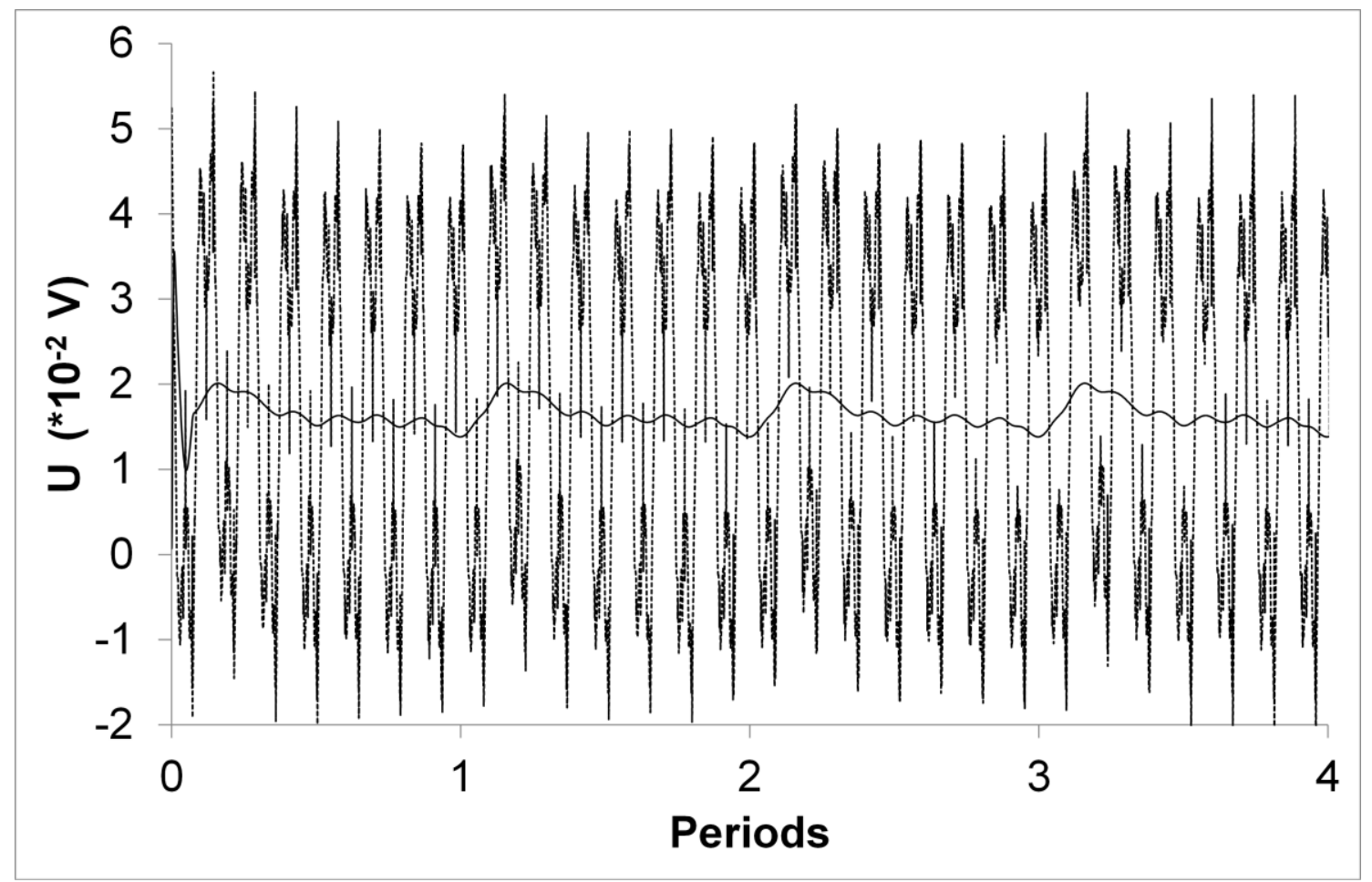

Fig. 1

Raw signal

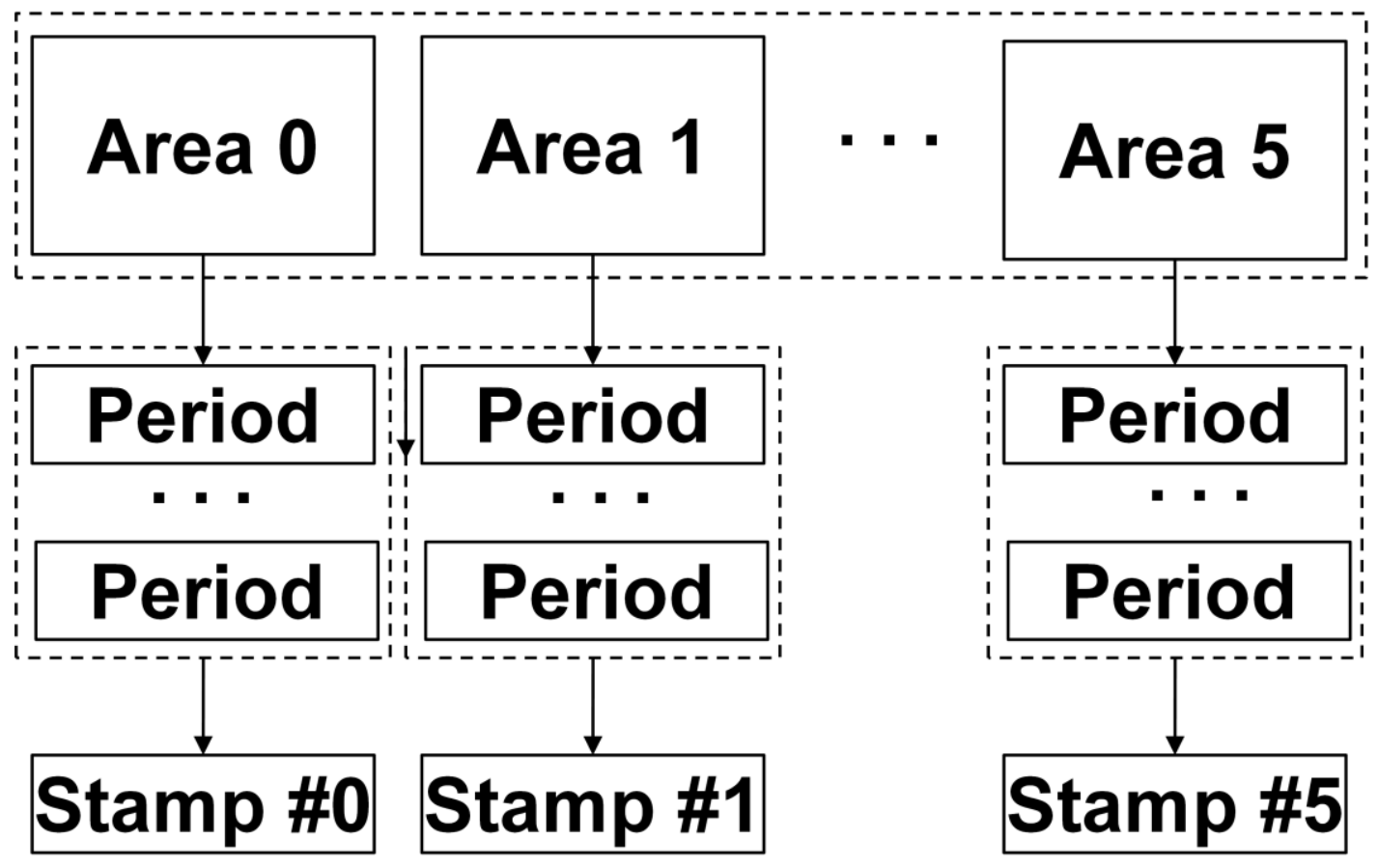

Fig. 2 


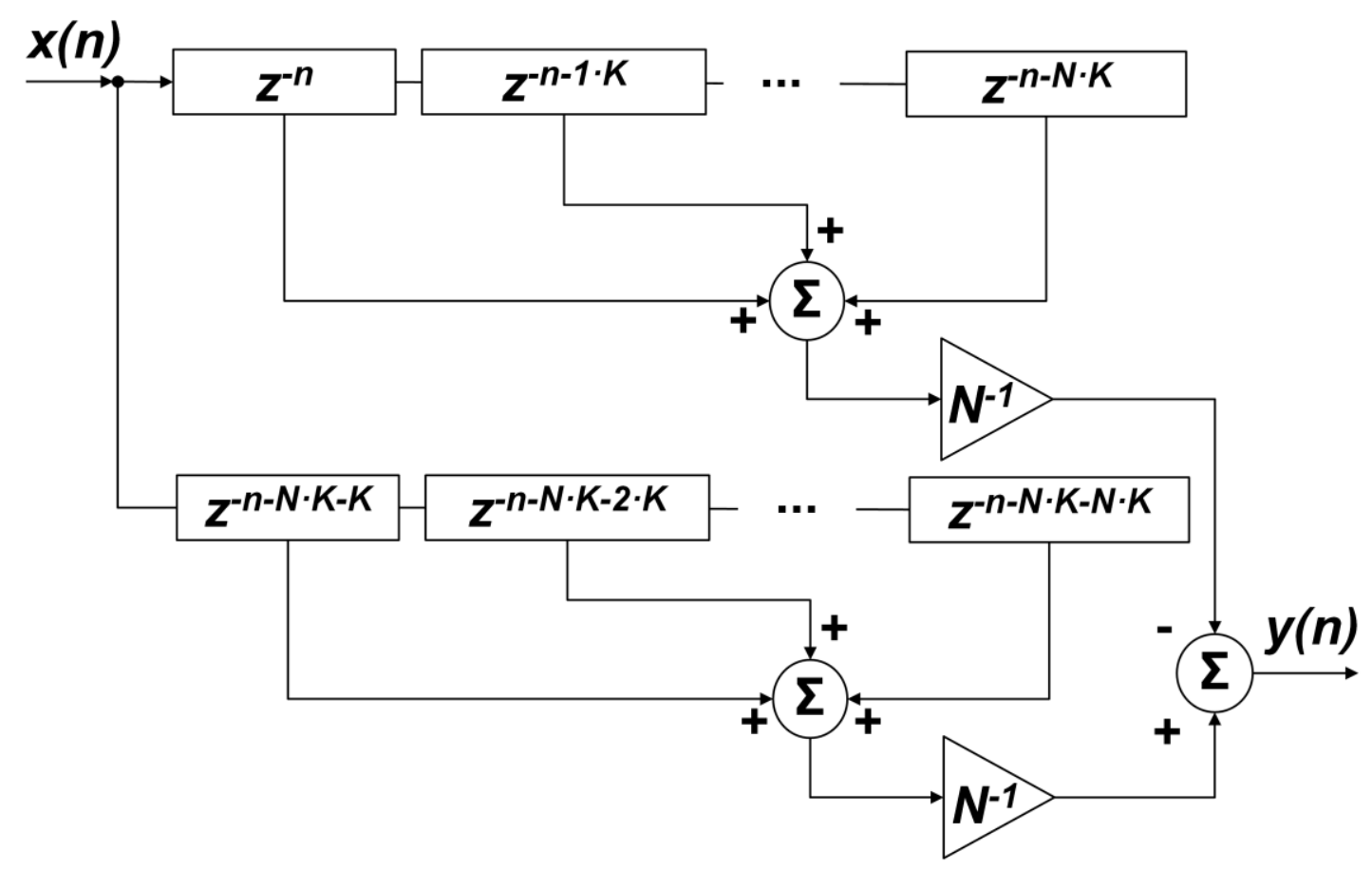

Fig. 3

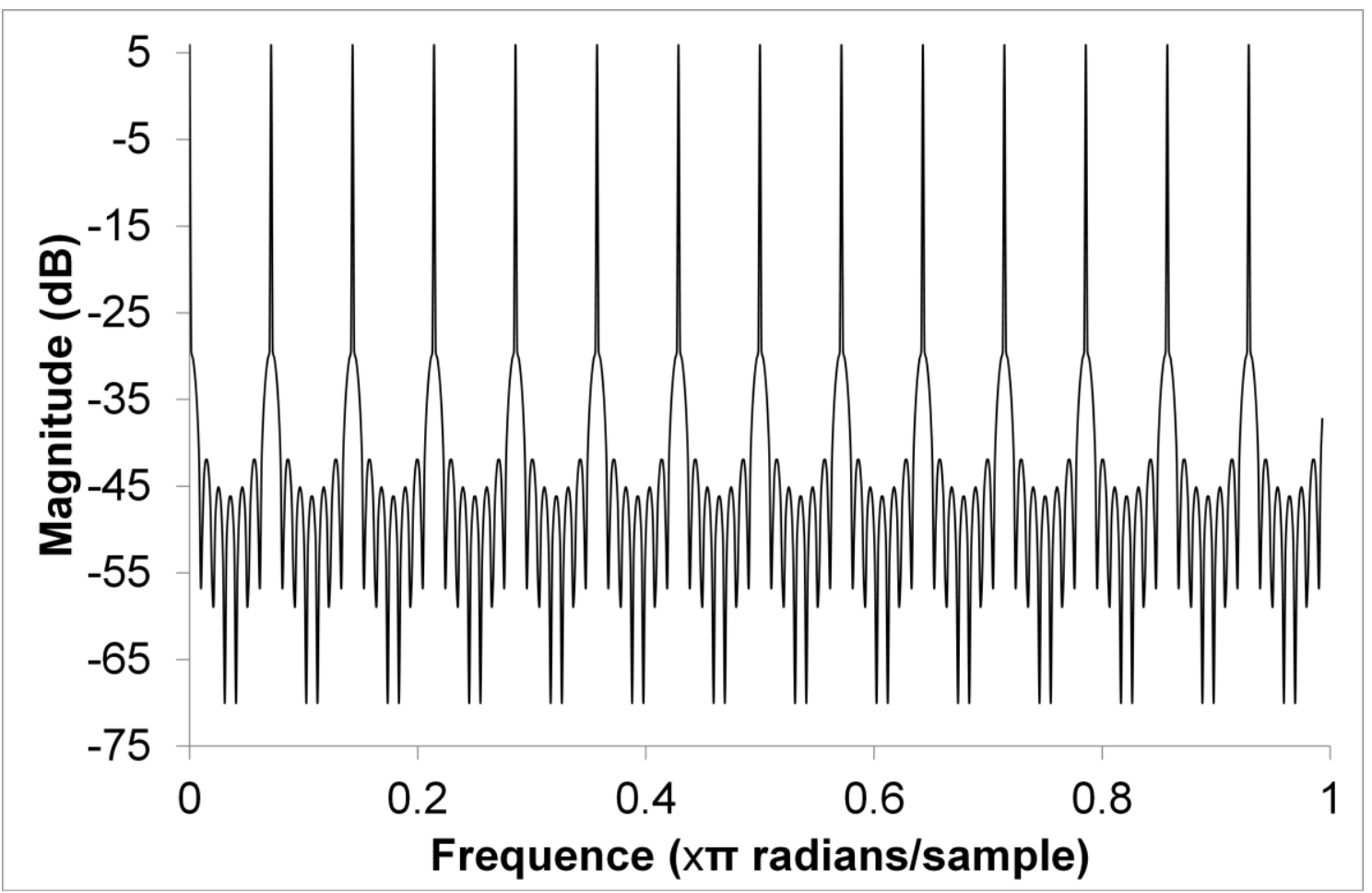

Fig. 4 


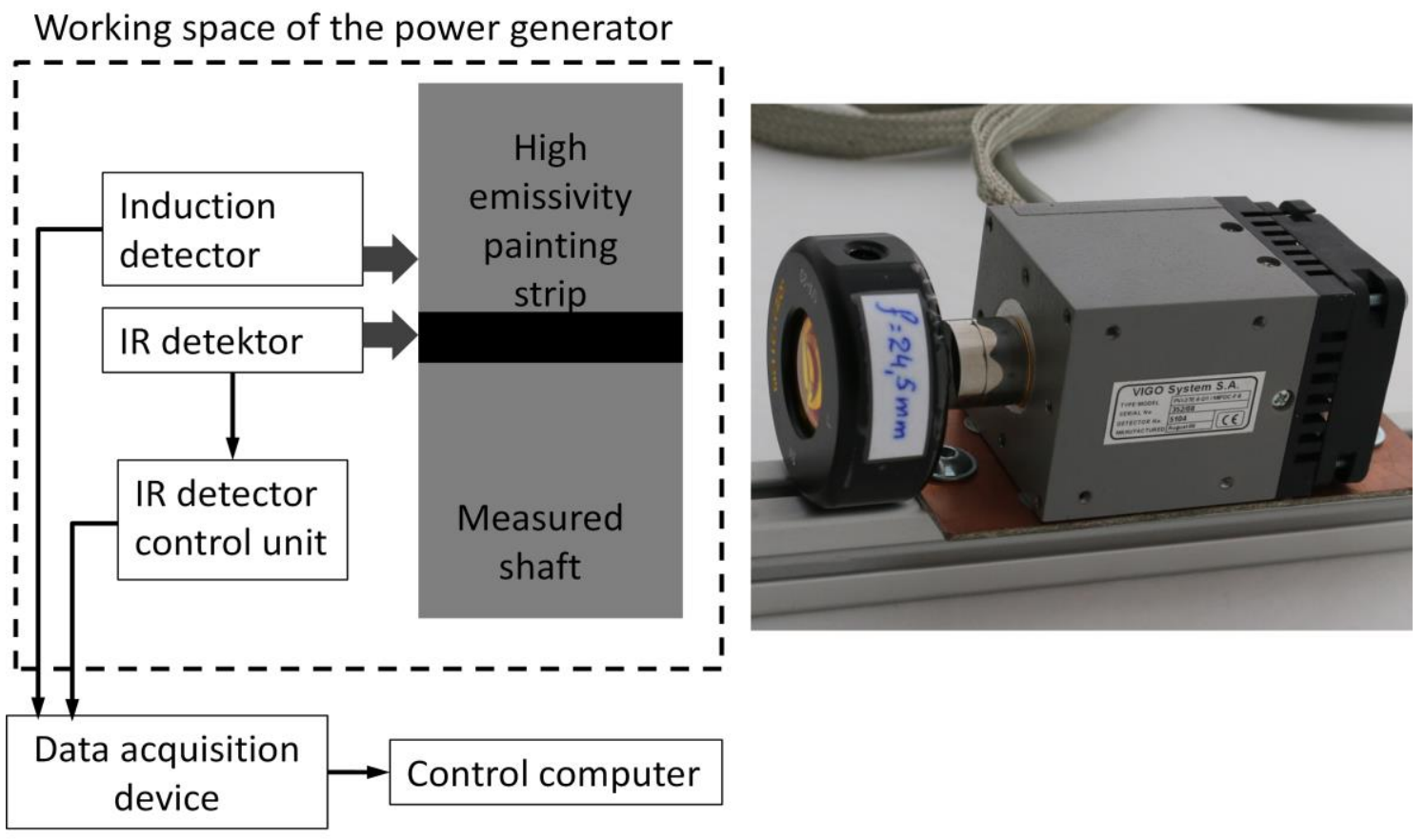

Fig. 5

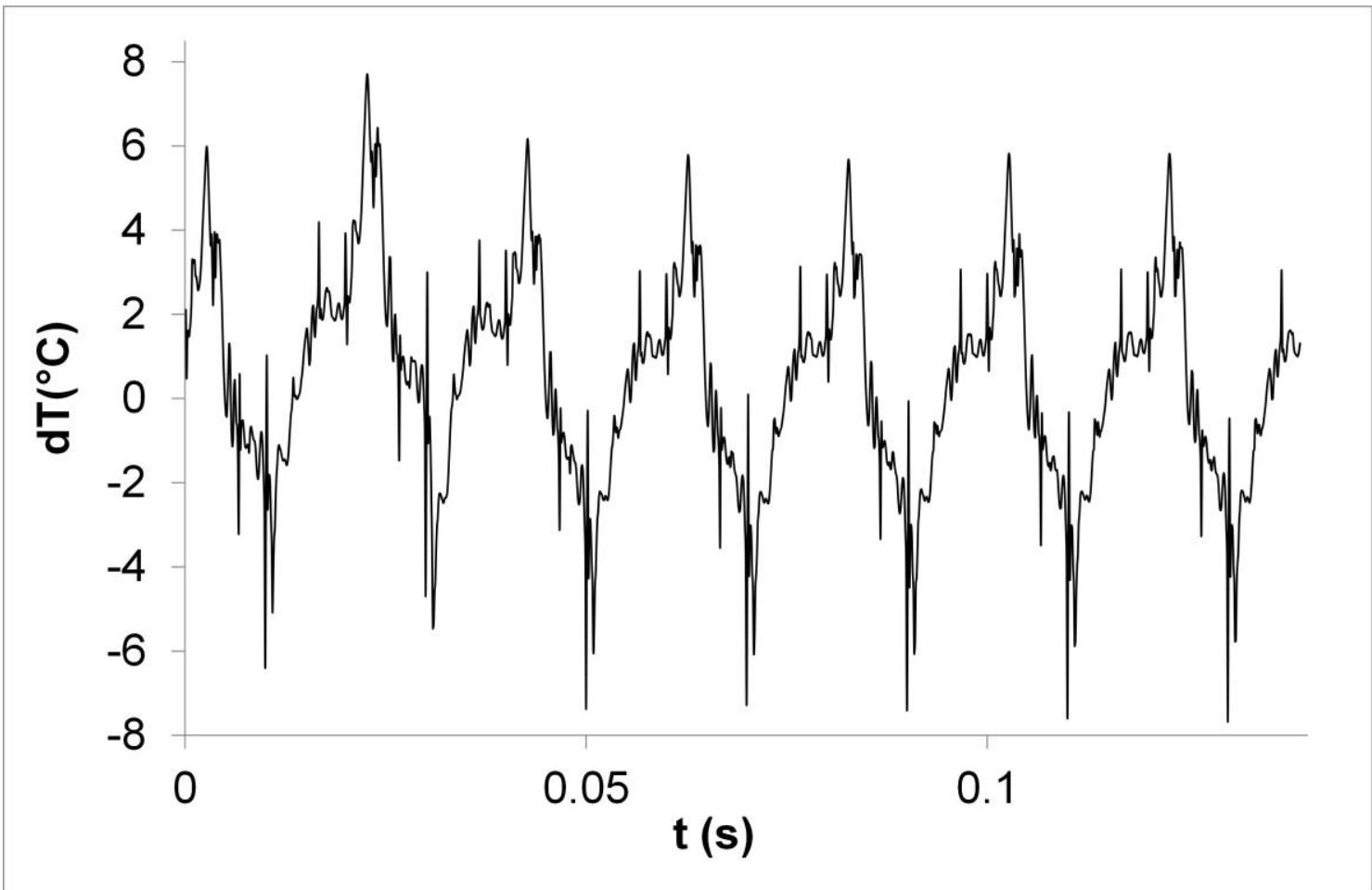

Fig. 6 


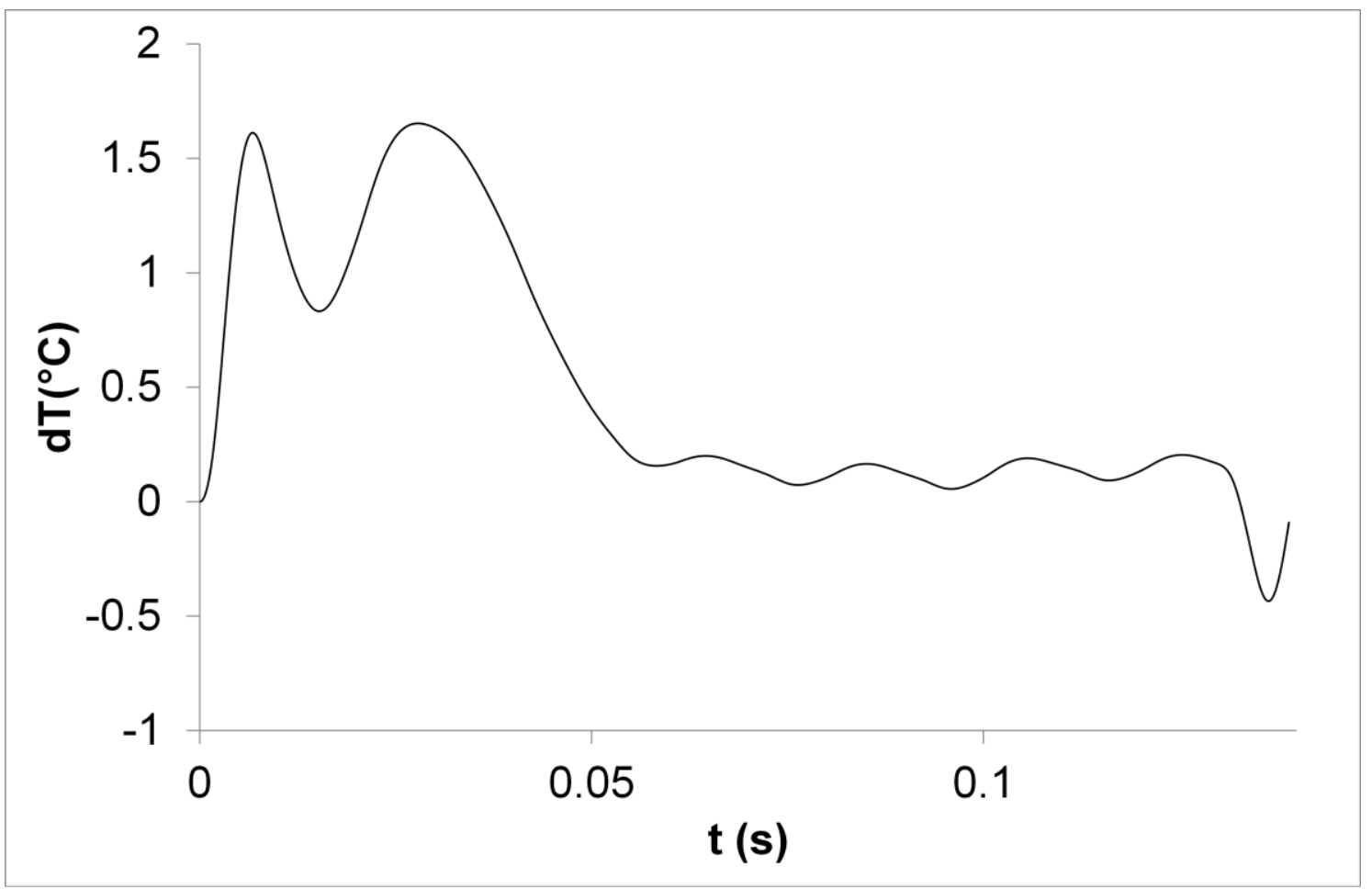

Fig. 7

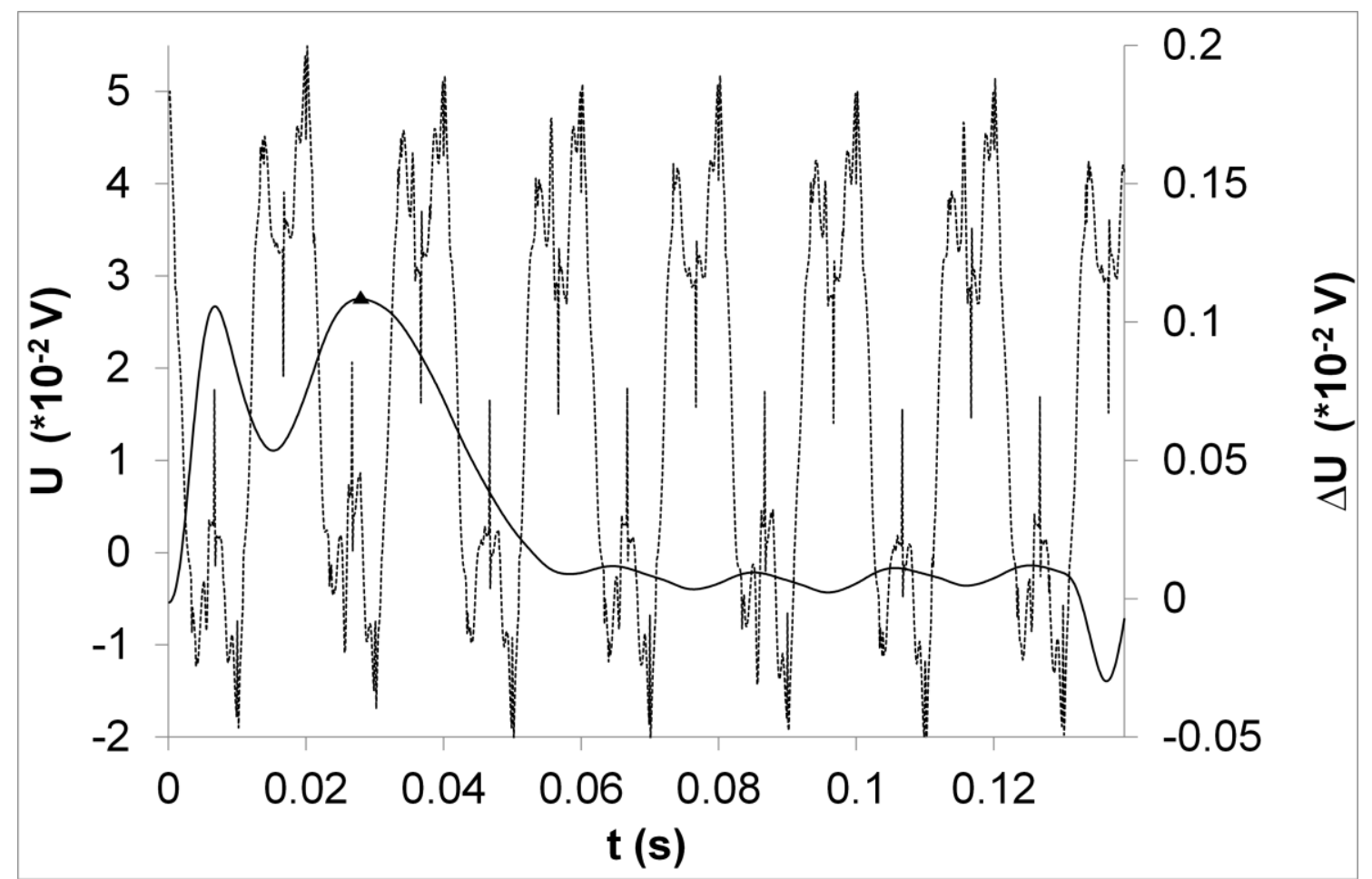

Fig. 8 


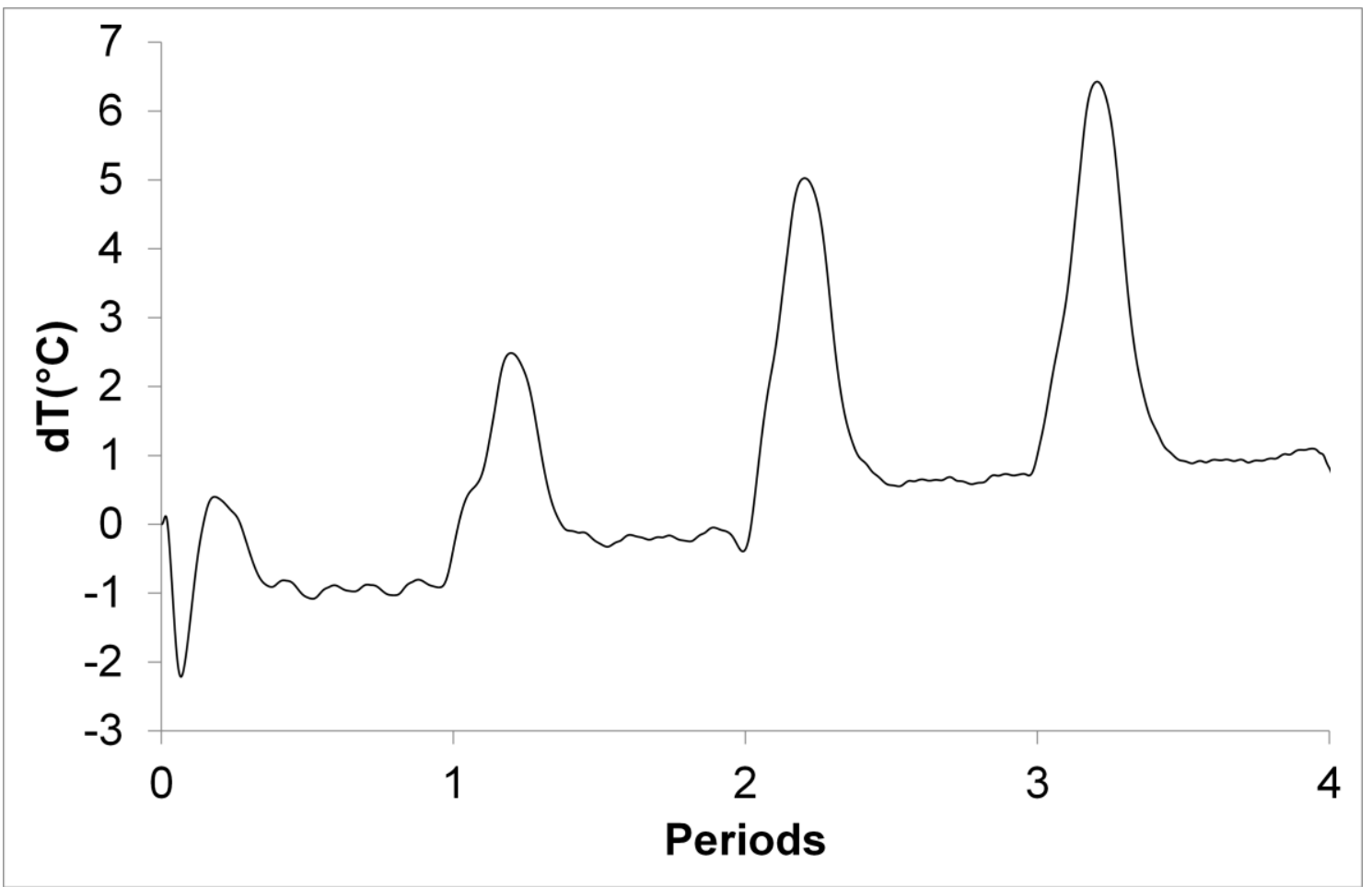

Fig. 9

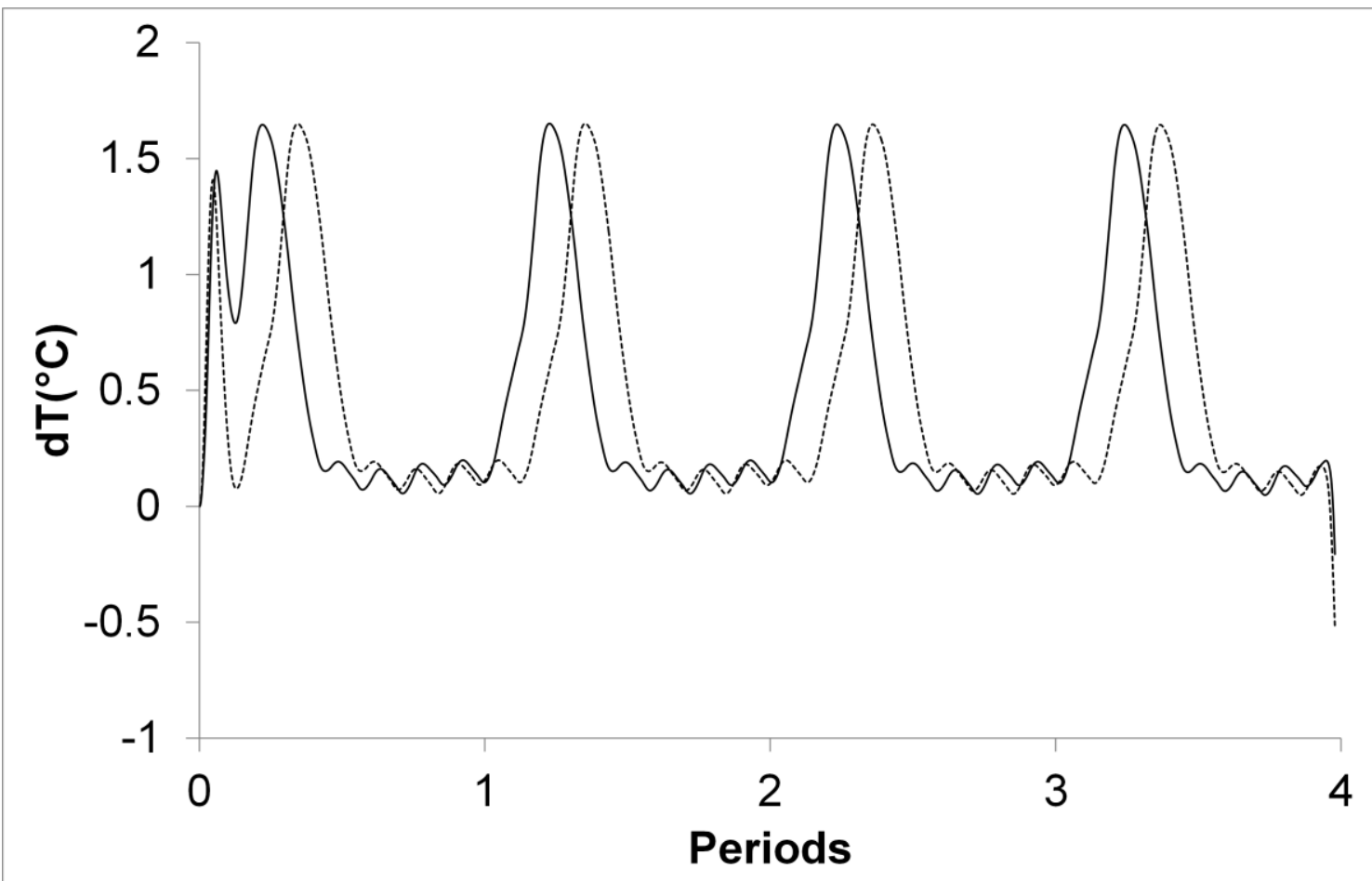

Fig. 10 


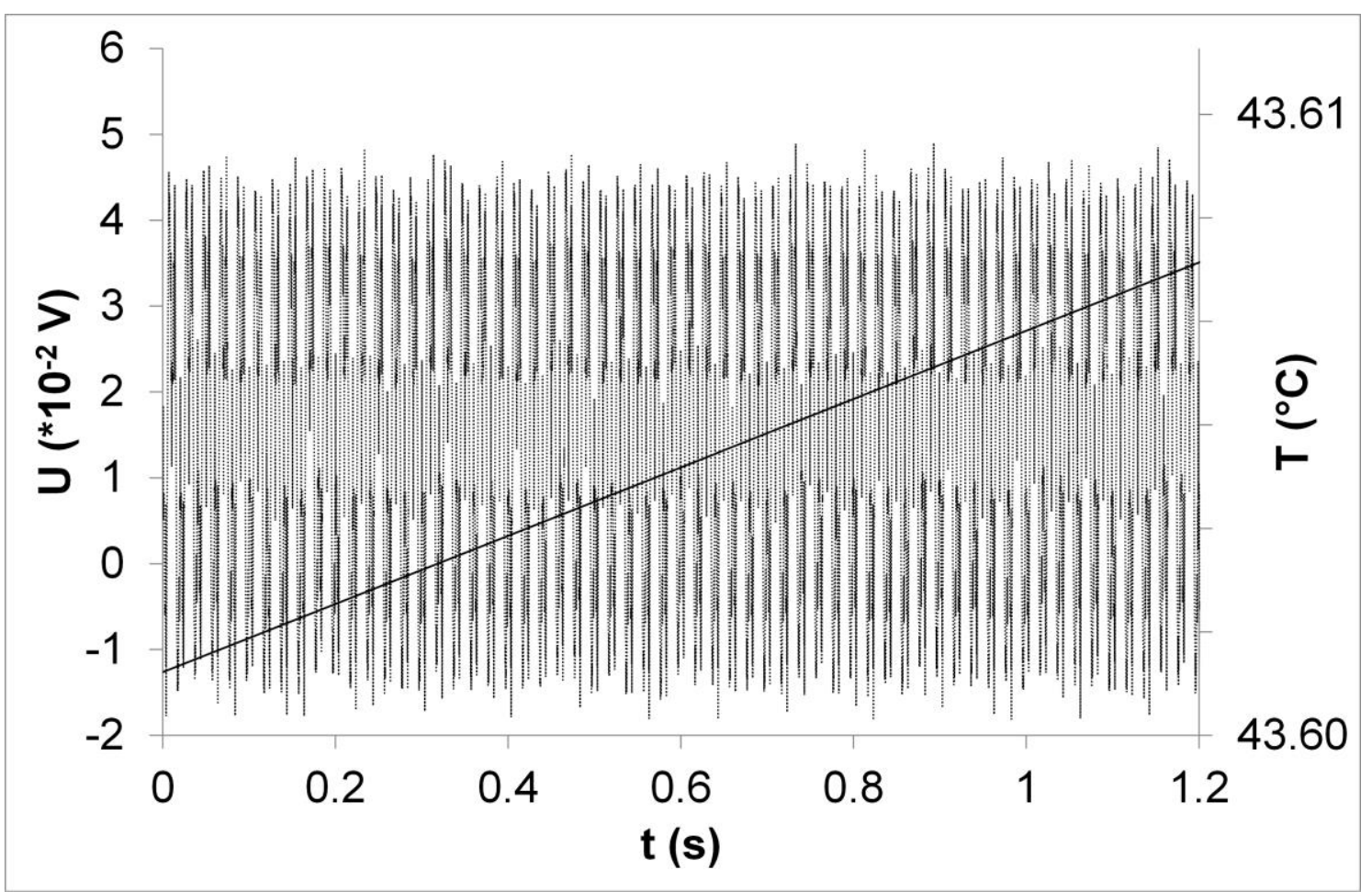

Fig. 11 